\title{
On the Effect of Time Delays in Negative Feedback Amplifiers
}

\author{
Luis Nero Alves, Rui L. Aguiar \\ Dept. Electrónica e Telecomunicações \\ Universidade de Aveiro/ Instituto de Telecomunicações \\ Aveiro, Portugal \\ nero@av.it.pt, ruilaa@det.ua.pt.
}

\begin{abstract}
Time delays are intrinsic to all existing devices and circuits. For the majority of applications, time delays are so small that their effects can be disregarded. However, when considering feedback amplifiers, depending on open-loop poles and loop gain, the effect of a small time delay inside the feedback path may turn to be of considerable importance. This paper analyzes the frequency response effects associated with time delays in feedback amplifiers, exploiting these effects to achieve bandwidth enhancements. An illustration of this effect is presented using a TLO82 in series-shunt configuration, attaining $100 \%$ bandwidth extension, when associated with a $36 m$ length $R G 58$ coaxial cable as delay element.
\end{abstract}

\section{INTRODUCTION}

Delayed feedback is a well established matter in some disciplines. For instance, in control systems modeling [1-3] time delay is a matter of fundamental importance: the control system used when guiding a missile (e.g.) must include a variable time delay in the feedback path, due to the communication delay between the missile and the controller. The effects of the delay on the feedback must be well predicted to prevent the risk of deviant behaviors (such as oscillations); for the above example this could signify missing or hitting the objective.

However, when discussing circuit design, the concept of delayed feedback has been usually disregarded. Although similar in nature, few contributions explore the effects of delays in feedback amplifiers [4-7]. Major emphasis of past achievements in this field point towards the necessity of careful design options to avoid stability problems brought by excess phase in the feedback path, such as avoiding large signal paths in the feedback when designing microwave amplifiers [4]. On [5, 6] the authors add a delay element to the feedback path of a voltage buffer to enhance rise time. On $[4,7]$ the presence of the delay element is analyzed as an excess phase element that can compromise stability.

Allowing some instability to arise is also a well known method of achieving bandwidth enhancements. In fact, the inclusion of a delay in the feedback path adds an excess phase to the loop promoting instability, but with careful control this can lead to bandwidth enhancements. Ultimately, even the presence of delay elements, intrinsic to active devices can be considered as a means of enhancing bandwidth, as well as another aspect to be careful in the design stage of very fast circuits.

In this paper the concept of delayed feedback is analyzed and applied to feedback amplifiers. Section 2 explores bandwidth enhancement as a result of the inclusion of a delay element on the feedback path. Several considerations concerning the presence of more than one pole in the feedback loop are also presented. Section 3 discusses stability restrictions of the proposed method. Section 4 presents a practical demonstration of the concept of bandwidth enhancement. Finally, section 5 draws some conclusions and points for future research.

\section{BANDWIDTH EXTENSION CONCEPT}

Figure 1 represents the general abstract model of a negative feedback amplifier with passive feedback (represented by the $\beta$ block). For analysis purposes we will separate the delay from the active elements. This way the discussion can be applied both to the case where the delay element is intentionally added and to the case where the delay is inherent to the active elements. Figure 1) represents the two possible cases: i) in fig. 1a) the delay appears on the feedback path - this may be the case of feedback amplifier with added delay; ii) in fig. 1b) the delay appears on the direct path, following the amplifier $A(s)$ - this, in turn, would

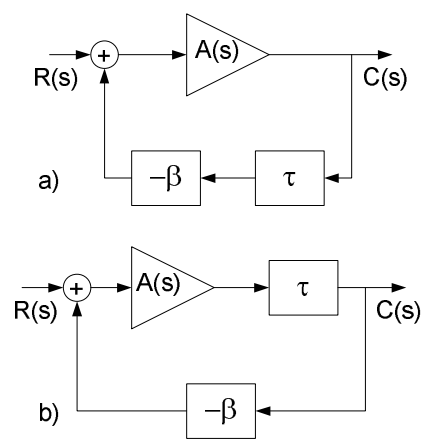

Figure 1- Feedback amplifier: a) with a delay on the feedback; b) with a delay on the direct path 
be the situation of an amplifier with intrinsic delay. Both the situations are realistic approaches of negative feedback amplifiers and both can be described in the same manner. The two control systems on fig. 1) have the same characteristic equation. Assuming that the amplifier $A(s)$ is accurately described by a transfer function having only poles (that is $A(s)=A_{o} / D(s)$, where $D(s)=0$ has roots with negative real part), the characteristic equation is given by,

$$
D(s)+G e^{-s \tau}=0
$$

where $G$ represents the loop gain given by $\beta A_{o}$, where $D(s)$ is a normalized polynomial in $\mathrm{s}$, such that $D(0)=1$. Furthermore, the two systems in fig. 1) also have the same closed-loop magnitude,

$$
\left|A_{f}(j w)\right|=\frac{A_{o}}{\left|D(j w)+G e^{-j w \tau}\right|}
$$

The only difference between them is the presence of the delay term on the numerator of $A_{f}(s)$ for the case in fig. 1b). Due to the presence of the exponential term in (2), finding analytical solutions for the cut-off frequency of a delayed feedback system is a difficult matter. Thus we resorted to numerical simulation procedures.

In order to compare cut-off frequencies between the system with delay and the system without delay, we used a three step numerical procedure:

i) The first step finds the cut-off frequency of the system without the delay, according to (3)

$$
\left|A_{f}\left(j w_{c}\right)\right|^{2}=\frac{A_{o}^{2}}{2(1+G)^{2}}
$$

Considering only one pole, the results of (3) are simply,

$$
w_{c}=(1+G) p_{1}
$$

2) The second step finds the cut-off frequency of the system including the delay, according to (5)

$$
\left|A_{f}\left(j w_{c d}\right)\right|^{2}=\frac{A_{o}^{2}}{2(1+G)^{2}}
$$

3 ) Finally on the third step the normalized cut-off frequency is computed according to equation (6).

$$
f_{c n}=\frac{w_{c d}}{w_{c}}
$$

$f_{c n}$ can also be interpreted as the relative gain in cut-off frequency between both systems, with and without delay. The same set of loop gains $(G)$ is assumed for all the three steps of the procedure. For the case of a simple first order system, $f_{c n}$ is the solution of the following implicit equation,

$$
\begin{aligned}
& \frac{2 G}{1+G}\left\{\frac{\cos \left[(1+G) f_{c n} p_{1} \tau\right]}{1+G}-f_{c n} \sin \left[(1+G) f_{c n} p_{1} \tau\right]\right\}= \\
& =2-\frac{1+G^{2}}{(1+G)^{2}}-f_{c n}^{2}
\end{aligned}
$$

Special care must be taken when finding the numerical solutions of (5). The exponential term can produce oscillatory behaviors on frequency response as shown on figure 2), producing multiple roots on equation (5). Here, the
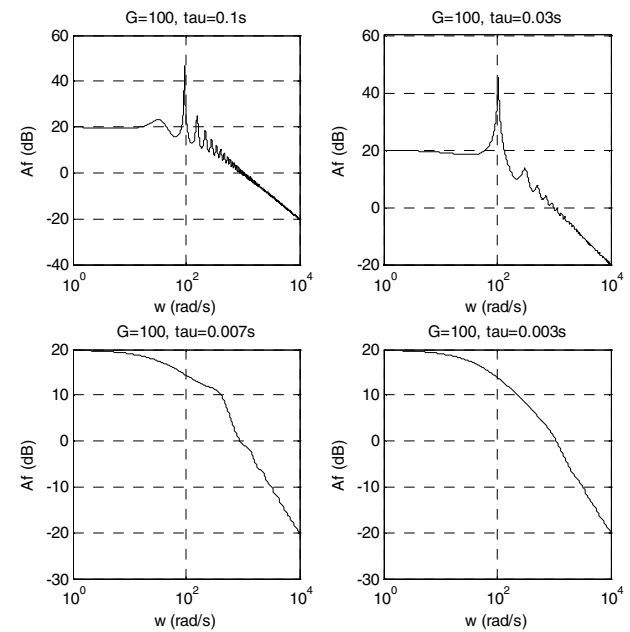

Figure 2 - Frequency response (magnitude) for different delays

cut-off frequency can be approximated as the first $-3 \mathrm{~dB}$ crossing of the system's frequency response.

Figure 2) shows frequency response plots according to equation (2), for several values of the time delay (assuming a normalized one pole transfer function with $p_{1}=1 \mathrm{rad} / \mathrm{s}$ and $A_{o}=1000$ ). Two observations can be made: i) the bandwidth increase is noticeable even for values of the time delay smaller than the amplifier time constant $\left(1 / w_{c} \approx 9,9 m s\right.$ with $G=100$ ); ii) frequency peaking (and associated oscillatory behaviors) appear for time-delays larger than the amplifier time constant.

Figure 3) shows plots of $f_{c n}$ for several values of time delay. As in figure 2), the suspected bandwidth enhancements are here clear. $f_{c n}$ shows a peaking behavior as a function of time delay, loop gain and pole frequency. This peaking behavior can be interpreted in the following way: as $G$ increases, decreasing the circuit's time constant, the relative gain in cut-off frequency also increases. This increase is maintained until the circuit's time constant (affected by the feedback) becomes comparable to the delay. Further increases on $G$ give rise to oscillatory behaviors, decreasing $f_{c n}$.

The plots of figure 3 show that $f_{c n}$ has almost constant maximums for systems with one pole and one delay on the feedback loop. This can be easily verified, assuming $G>>1$

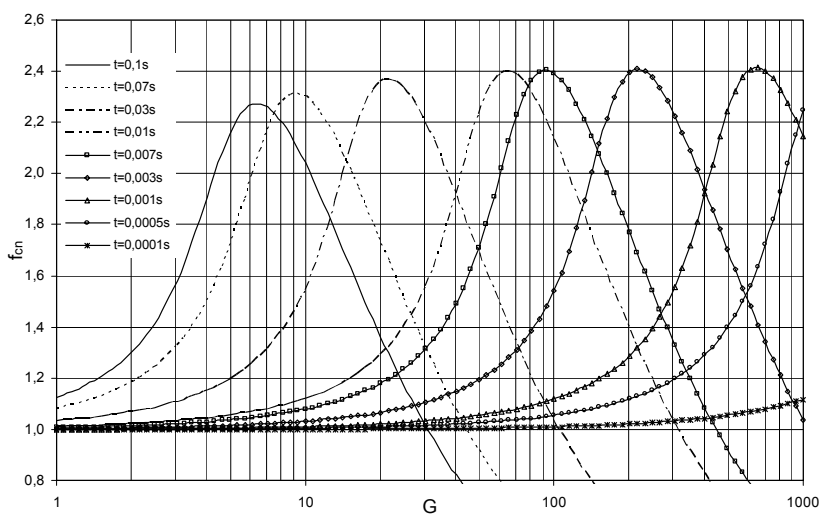

Figure 3 - Normalized cut-off frequency as function of the loop gain (for various delay values) 


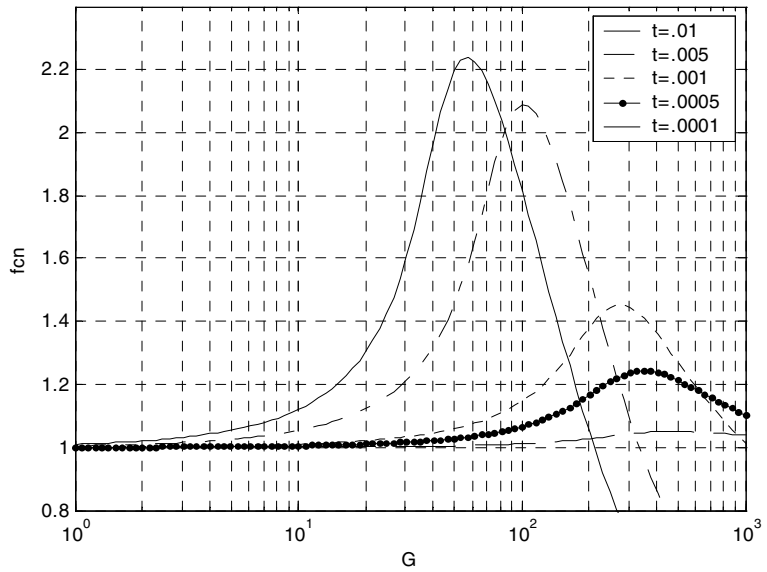

Figure 4 - Normalized cut-off frequency of a second order system with delay, for various delay values.

and taking the zero off the first derivative of (7). The approximate solution for the maximum is given by (8).

$$
\left\{\begin{array}{c}
f_{c n_{\max }}=1+\sqrt{2} \approx 2.4142 \\
G_{\max }=\frac{\pi}{2 p_{1} \tau f_{c n_{\max }}} \approx \frac{0.6506}{p_{1} \tau}
\end{array}\right.
$$

According to equation (8) the maximum attainable cut-off frequency gain is 2.414, representing an extension in bandwidth of $141.4 \%$. However, there are several factors that limit this maximum gain in reality, namely: i) stability limitations - as shown in figure 2 oscillatory behaviors may arise; ii) open loop gain $\left(A_{0}\right)$ - a natural limitation on the loop-gain; iii) the presence of more than one pole - posing limitations on the maximum gain and on stability; and, iv) the real delay element - pure delays are difficult to synthesize as desired.

\section{A. Higher order systems with delay}

The analysis of higher order systems can be done in similar manner as before. Figure 4) and 5) shows $f_{c n}$ plots, for a system with two poles and delay. The first pole was assumed to be dominant, and placed at the frequency of $1 \mathrm{rad} / \mathrm{s}$. The second pole occurs at a frequency of $d \mathrm{rad} / \mathrm{s}$. The plots on figure 4) show $f_{c n}$ for several values of the delay, with fixed pole separation $(d=100)$. As before, the presence of the delay element in the feedback loop produces a peaking behavior. However, the amplitude of the peaks decreases with increasing loop gains. Figure 5) shows $f_{c n}$ plots for various values of the pole separation constant, $d$, with fixed delay $(\tau=0.01 s)$. When the two poles are closely placed, the peaking effect is negligible; this is a major limitation for the maximum attainable bandwidth gain, in real systems.

\section{Stability Restrictions}

A feedback system with one or two poles characteristic equation is always stable under negative feedback constraints, as long as the poles have negative real parts. The root-locus of a second order system has two branches beginning on the poles and splitting vertically to infinity from the medium point between them (if both poles are real, the splitting occurs on the real axis). The presence of a delay

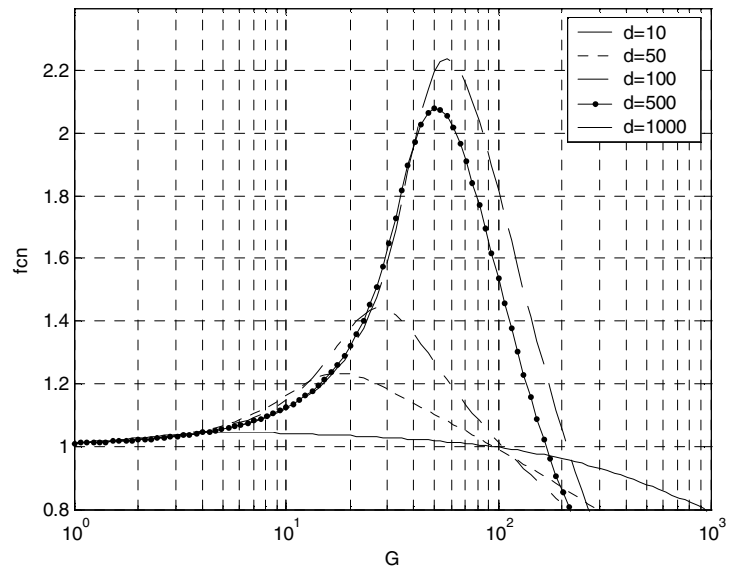

Figure 5- Normalized cut-off frequency of a second order system with delay, for various pole separation values.

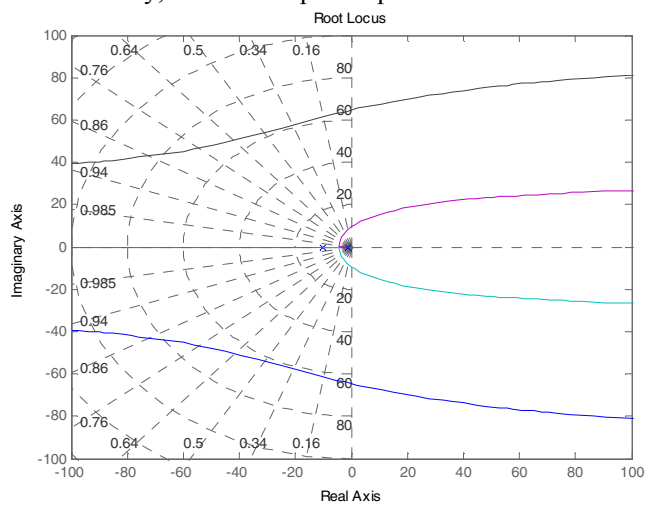

Figure 6- Root locus for a system with two poles and delay (magnified sketch near the origin).

in a second order system generates an infinite number of branches that cross the imaginary axis through the positive real side of the complex plane (a magnified sketch near the origin is presented in figure 6). The original branches of the second order system (those beginning on the system poles) are bent towards the positive side of $s$ (see figure 6).

The effect of the delay is to promote instability on the overall system (this is also true for a first order system). However the system behavior is primarily ruled by the system open loop poles (as these are the roots that originate the critical branches), as long as the delay is small enough in order to have sufficient separation between the secondary branches.

\section{A. Marginal Stability}

Marginal stability conditions limit the maximum realizable loop-gain, and consequently the maximum bandwidth benefit in delayed feedback systems.

In a delayed feedback system with only poles inside the feedback loop, the characteristic equation is expressed by (1). Using $s=x+j y$, the characteristic equation can be written,

$$
\left\{\begin{array}{l}
D_{r}(x, y)+G e^{-x \tau} \cos (y \tau)=0 \\
D_{i}(x, y)-G e^{-x \tau} \sin (y \tau)=0
\end{array}\right.
$$

Where $D_{r}(x, y)$ and $D_{i}(x, y)$ are respectively, the real and imaginary parts of $D(s)$. Marginal stability can be evaluated through the points where the root-locus crosses the 
imaginary axis. These intersections are simply the solutions of (9) setting $x=0$, resulting in the following (where $y_{0}$ are the imaginary axis intercept points),

$$
\begin{gathered}
\tan \left(y_{0} \tau\right)=-\frac{D_{i}\left(0, y_{0}\right)}{D_{r}\left(0, y_{0}\right)} \\
G=\frac{D_{i}\left(0, y_{0}\right)}{\sin \left(y_{0} \tau\right)}
\end{gathered}
$$

Equation (10) gives information on the oscillating frequencies, while equation (11) provides the necessary loopgain to achieve marginal stability. As can be seen in (10), finding $y_{0}$ is a numerical process, with no immediate analytical solution (except for cases where the quotient of $D_{i}$ to $D_{r}$ is a constant value). To prevent oscillations is sufficient to adjust $G$ with values below those predicted by equation (11). Table (1) summarizes the forms of equations (10) and (11) for systems of orders 1, 2 and 3.

\section{TABLE I. TABLE 1 MARGINAL STABILITY CONDITIONS}

\begin{tabular}{|c|c|c|c|}
\hline $\boldsymbol{n}$ & $\tan \left(y_{0} \tau\right)$ & $\boldsymbol{G}$ & Notes \\
\hline 1 & $-\frac{y_{0}}{p_{1}}$ & $\frac{y_{0}}{p_{1} \sin \left(y_{0} \tau\right)}$ & \\
\hline 2 & $\frac{A y_{0}}{y_{0}^{2}-P}$ & $\frac{A y_{0}}{P \sin \left(y_{0} \tau\right)}$ & $\begin{array}{l}A=p_{1}+p_{2} \\
P=p_{1} p_{2}\end{array}$ \\
\hline 3 & $\frac{y_{0}^{3}-A_{2} y_{0}}{A_{3}-A_{1} y_{0}^{2}}$ & $y_{0} \frac{A_{2}-y_{0}^{2}}{A_{3} \sin \left(y_{0} \tau\right)}$ & $\begin{array}{l}A_{1}=p_{1}+p_{2}+p_{3} \\
A_{2}=p_{1} p_{2}+p_{1} p_{3}+p_{2} p_{3} \\
A_{3}=p_{1} p_{2} p_{3}\end{array}$ \\
\hline
\end{tabular}

\section{EXPERIMENTAL RESULTS}

This bandwidth enhancement concept was analyzed in a real circuit, using an TL082 operational amplifier (as in figure $7)$ ). We used as delay element a variable length transmission line (TL), consisting of 3 to 36 meters of standard $R G 58 \mathrm{U} / \mathrm{C}$ $50 \Omega$ cable. In order to have a perfect delay, the TL needs to be correctly matched (even for small frequencies). This was done on both ends of the TL; on the source, a $50 \Omega$ match was imposed by the unit gain buffer $A_{1}$; and on the load by a similar buffer $\left(A_{2}\right)$ with $50 \Omega$ input impedance. These buffers are simple class $\mathrm{AB}$ output stages with large bandwidth (at least 2 orders than the expected system bandwidth).

Figure 8) shows the experimental results for various TL lengths. The solid lines represent polynomial fitting curves for each data set. The similarity with the theoretical results on figure 4) is evident, where larger time delays (larger TL lengths) produce large bandwidths at lower loop-gains. Deviations from the fitting curves may arise due to modeling approximations used for these devices and TL termination aspects.

\section{CONCLUSIONS}

A new concept for the bandwidth enhancement has been presented. The presence of time delays in feedback

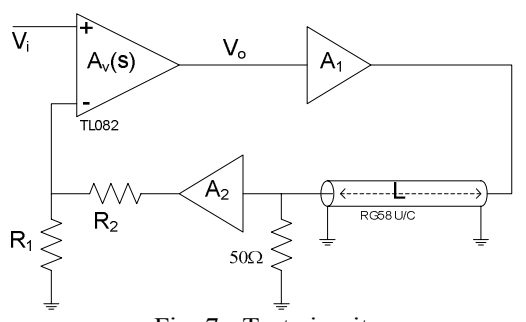

Fig. 7 - Test circuit

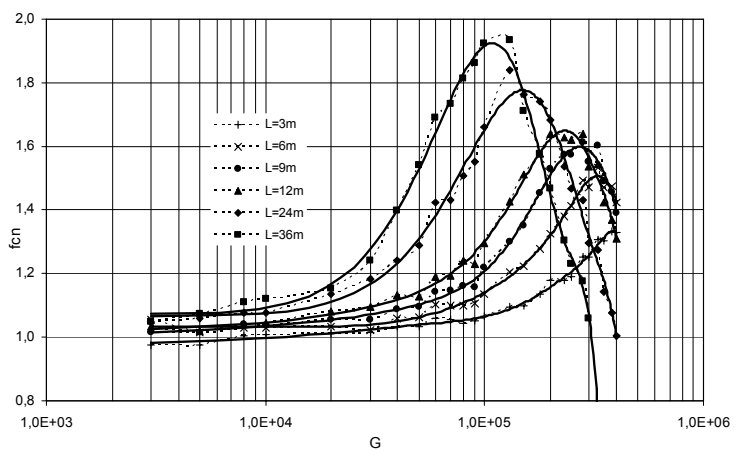

Fig. 8 - Normalized cut-off frequency (for various transmission-line lengths)

amplifiers may be explored as a benefit, which can lead to bandwidth extensions as high as the theoretical maximum of $141.4 \%$. Experimental results attain a maximum of almost $100 \%$, using common discrete electronic components. Better results can be achieved using custom designed integrated circuits, with simulated delays (both passive and active implementations).

The application of this concept to high frequency design is of major interest - in GHz bandwidth amplifiers, small TLs feasible in actual IC technologies, may easily produce the delays required for large bandwidth improvements.

\section{REFERENCES}

[1] J. S. Freudenberg, D. P. Looze, "A Sensitivity Tradeoff for Plants with Time Delay". IEEE Trans. on Automatic Control, Feb. 1987.

[2] G. J. Silva, A. Datta, S. P. Bhattacharyya, "Stabilization of Time Delay Systems". Proc. American. Control Conf., Chicago, Illinois, Jun. 2000.

[3] G. J. Silva, A. Datta, S. P. Bhattacharyya, "Determination of Stabilizing Feedback Gains for Second-order Systems with Time Delay". Proc. American. Control Conf., Arlington, Virginia, Jun. 2001.

[4] G. Nowack, "Time-Delays in Microstructures and Stability-Problems in Ultra-High Speed Amplifiers for Optical WANs", TELSIKS'99, Nis, Yugoslavia, Oct. 1999.

[5] L. Weiss, "Achieving Subnanosecond Delays Using Feedback with the Current Switch", IEEE Journal Solid-State Circ., Dec. 1966.

[6] E. K. Reedy, J.F. Pierce, "A Complementary Driver with a Subnanosecond Rise Time", IEEE Journal Solid-State Circ., Vol. sc1, No 2, Oct. 1969.

[7] F. H. Blecher, "Design Principles for Single Loop Transistor Feedback Amplifiers", IRE Trans. on Circ. Theory, Sep. 1957. 